\title{
Simultaneous infrared-ultrasound irradiation in organic synthesis: Acylation of amines, alcohols and amino alcohols
}

Ricardo Alfredo Luna-Mora, ${ }^{1}$ Fernando Ortega-Jiménez, ${ }^{1}$ Hulme Ríos-Guerra, ${ }^{1}$ José Guadalupe García-Estrada, ${ }^{1}$ Francisco Javier Pérez-Flores, ${ }^{2}$ Jessica González-Carrillo, ${ }^{1}$ Ángeles Torres-Reyes, ${ }^{1}$ Linda Moreno-González, ${ }^{1}$ Alejandro MartínezZaldivar, ${ }^{1}$ José Guillermo Penieres-Carrillo. ${ }^{*}$

${ }^{1}$ Facultad de Estudios Superiores Cuautitlán-Universidad Nacional Autónoma de México, Sección de Química Orgánica, FESC, Campo 1, Cuautitlán Izcalli, C. P. 54740, México.

${ }^{2}$ Instituto de Química-Universidad Nacional Autónoma de México, Circuito Exterior, Ciudad Universitaria, Ciudad de México, C.P. 04510, México.

*Corresponding autor: José Guillermo Penieres-Carrillo, Email: penieres@unam.mx . Facultad de Estudios Superiores Cuautitlán-Universidad Nacional Autónoma de México, Sección de Química Orgánica, FESC, Campo 1, Cuautitlán Izcalli, C. P. 54740, México.

Received September 26 ${ }^{\text {th }}, 2018$; Accepted April 11 $1^{\text {th }}, 2019$.

DOI: http://dx.doi.org/10.29356/jmcs.v63i2.692

\begin{abstract}
The acylation of both aliphatic and aromatic amines, alcohols and amino alcohols by simultaneous infraredultrasound irradiation (SIUI) in solvent-free conditions in short reaction times and with good to excellent yields was achieved. The results obtained with SIUI and for thermal, infrared and ultrasound energy sources are compared. This is the first report regarding to the application of SIUI in acylation reactions.
\end{abstract}

Keywords: Acylation reactions; Green chemistry; simultaneous infrared-ultrasound irradiation (SIUI).

Resumen. Se realizó la acilación de aminas, alcoholes y amino alcoholes alifáticos y aromáticos mediante la irradiación simultánea de energía de infrarrojo y de ultrasonido (SIUI), en ausencia de disolventes, en tiempos de reacción cortos y con excelentes rendimientos de reacción. Los resultados obtenidos con SIUI fueron comparados con los obtenidos mediante el empleo de energía térmica, de infrarrojo y de ultrasonido. Este es el primer informe relacionado a la aplicación de SIUI en reacciones de acetilación.

Palabras clave: Reacciones de acilación; Química Verde; irradiación simultánea infrarrojo-ultrasonido (SIUI).

\section{Introduction}

Acylation is one of the most used reactions in organic synthesis [1-4] as a protecting group of amine, alcohol, and thiol functional groups or as a functional group of the final structure of a product.

Many variations of this important reaction are known, such as in acetylsalicylic acid, which was introduced to clinical use by Dreser in 1899 and is still used as an analgesic around the world. [5,6] Acylation reactions are used in carbohydrate chemistry [3,7] and to improve stability for many compounds, [8] such as nucleosides, [9] steroids [10] and natural products. [11]

A general method for acylation reactions is the use of acetic anhydride at either reflux or room temperature with the appropriate substrate, under homogeneous or heterogeneous media, [12] with or without the use of a catalyst. [13,14] Acetyl chloride, [15] acetic acid [16] or acyl urea [17] can also be employed. Numerous other strategies have been used, such as with a Lewis acid, [18-23], solid supports [24-26] and polymeric acids. [27] In many cases, a solvent is used, such as benzene, toluene, and xylenes, [28,29] and also in solvent-free conditions, [30,31] with the use of microwave irradiation, [32] ultrasound, [33] infrared, [34] ionic liquids, [35-37] and enzymes. [38,39] Actually the use of a base like triethylamine or pyridine, in stoichiometric amounts, it's the most common reaction condition for acylation reactions.

One of the so-called non-classical energy forms includes infrared irradiation. This method of promoting a reaction has the advantages of minimizing reaction time, improve the product yield and avoid undesired byproducts and is a direct 
form of heating to activate a reaction. Therefore, the infrared irradiation has found a vast field of application in organic synthesis. [40]

The use of alternative energy sources for the activation of chemical reactions is well known and simultaneous microwave-ultrasound (SMUI) is a good example of the combination of energy sources resulting in the enhancement in reaction rates by integration of the technologies based on enhanced heat and mass transfer. [41,42]

The combination of these energy sources results in the improvement of reactions yields and reaction times that go from hours by conventional thermal heating to seconds by SMUI. [43-50]

In this paper, we present a new methodology to perform acylation reactions of a series of anilines, alcohols, and amino alcohols by simultaneous infrared-ultrasound irradiation (SIUI) in solvent-free conditions and without the use of any catalyst. Besides, these results with other energy sources are compared.

\section{Experimental Section}

IR reactions were irradiated at the wavelength of $1100 \mathrm{~nm}\left(9.09 \mathrm{~cm}^{-1}\right)$ using a home-made device adapted with a THERA-TERM OSRAM infrared bulb of $250 \mathrm{~W} / 125 \mathrm{~V}$ and a capacitor to control temperature. [52] Ultrasound experiments were carried out in a Cole Parmer $500 \mathrm{~W} / 115 \mathrm{~V}$ instrument with temperature control and with an irradiation frequency of 20 KHz. For SIUI IR and US, instruments were used: IR at $10 \mathrm{~cm}$ from the reaction vessel and employment a rheostat at $80 \%$; US with $1.4 \mathrm{~cm}$ of the probe horn located in the reaction mixture and $50 \%$ of amplitude, Fig. 1.

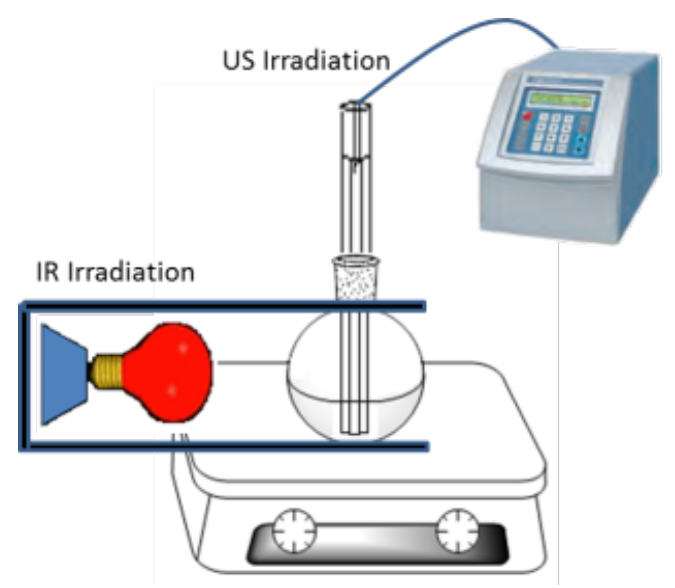

Fig. 1. SIUI apparatus.

\section{Procedure and spectral data}

General procedure. A mixture of amine, alcohol or amino alcohol $(1.0 \mathrm{mmol})$ and an acylating agent $(1.4 \mathrm{mmol})$ with the respective energy by the time shown in the corresponding table was irradiated until substrate was completely converted to the acylated product. Then, to the mixture reaction was added ice water and the precipitated product was filtered under vacuum and dried. Selected spectral data (spectral data of all obtained compounds in Supporting Information file are presented).

$\mathrm{N}$-Cyclohexylacetamide (I 1 / II 1) [53]. White solid, m.p. 101.5-102.3 ${ }^{\circ} \mathrm{C}, 54.4 \mathrm{mg}, 99 \%, \mathrm{Rf}=0.60$ (Hex-EtOAc 6:4). ${ }^{1} \mathrm{HNMR}\left(300 \mathrm{MHz}, \mathrm{CDCl}_{3}\right): \delta 5.89$ (br s, $\left.1 \mathrm{H}, \mathrm{NH}\right), 3.67-3.73(\mathrm{~m}, 1 \mathrm{H}), 1.92(\mathrm{~s}, 3 \mathrm{H}), 1.85-1.90(\mathrm{~m}, 2 \mathrm{H}), 1.65-1.69(\mathrm{~m}, 2 \mathrm{H})$, 1.56-1.60 (m, $1 \mathrm{H}), 1.26-1.35(\mathrm{~m}, 2 \mathrm{H}), 1.05-1.15(\mathrm{~m}, 3 \mathrm{H}) .{ }^{13} \mathrm{CNMR}\left(75 \mathrm{MHz}, \mathrm{CDCl}_{3}\right): \delta 169.2,48.2,33.1,25.5,24.9,23.5$.

$N$-Benzylacetamide (I 2 / II 2) [54]. White solid, m.p. 61-63 ${ }^{\circ} \mathrm{C}, 283 \mathrm{mg}, 99 \%, \mathrm{Rf}=0.37$ (Hex-EtOAc 6:4). ${ }^{1} \mathrm{HNMR}$ (300 $\left.\mathrm{MHz}, \mathrm{CDCl}_{3}\right): \delta 7.34-7.22(\mathrm{~m}, 5 \mathrm{H}), 6.14(\mathrm{~s}, 1 \mathrm{H}, \mathrm{NH}), 4.39$ (d, $\left.J=4.4 \mathrm{~Hz}, 2 \mathrm{H}\right), 1.98(\mathrm{~s}, 3 \mathrm{H}) .{ }^{13} \mathrm{CNMR}\left(75 \mathrm{MHz}, \mathrm{CDCl}_{3}\right): \delta$ $170.0,138.3,128.6,127.8,127.4,43.7,23.2$.

$N$-Phenylacetamide (I 4 / II 4) [55]. White solid, m.p. 111-113 ${ }^{\circ} \mathrm{C}, 130.95 \mathrm{mg}, 97 \%$, Rf $=0.53$ (Hex-EtOAc 5:5). ${ }^{1} \mathrm{HNMR}$ $\left(300 \mathrm{MHz}, \mathrm{DMSO}_{-d 6}\right): \delta 8.5(\mathrm{~s}, 1 \mathrm{H}), 7.51(\mathrm{~d}, J=7.8 \mathrm{~Hz}, 2 \mathrm{H}), 7.27(\mathrm{t}, J=7.8 \mathrm{~Hz}, 2 \mathrm{H}), 7.07(\mathrm{t}, J=7.5 \mathrm{~Hz}, 1 \mathrm{H}), 2.12(\mathrm{~s}, 3$ H). ${ }^{13} \mathrm{CNMR}\left(75 \mathrm{MHz}, \mathrm{DMSO}-d_{6}\right): \delta 169.1,137.9,128.7,124.1,120.1,24.2$. 
$\mathrm{N}$-(2-Bromophenyl)acetamide (I 7 / II 7) [56]. White solid, m.p. 97-99 ${ }^{\circ} \mathrm{C}, 236 \mathrm{mg}, 99 \%, \mathrm{Rf}=0.49$ (Hex-EtOAc 5:5). ${ }^{1} \mathrm{HNMR}\left(300 \mathrm{MHz}, \mathrm{CDCl}_{3}\right): \delta 8.25(\mathrm{~d}, J=8.3 \mathrm{~Hz}, 1 \mathrm{H}), 7.51(\mathrm{~d}, J=23.4 \mathrm{~Hz}, 1 \mathrm{H}), 7.49-7.41(\mathrm{~m}, 1 \mathrm{H}), 7.28-7.20(\mathrm{~m}, 1 \mathrm{H})$, $6.90(\mathrm{t}, J=7.7,1.6 \mathrm{~Hz}, 1 \mathrm{H}), 2.16(\mathrm{~s}, 3 \mathrm{H}) .{ }^{13} \mathrm{CNMR}\left(75 \mathrm{MHz}, \mathrm{CDCl}_{3}\right): \delta 168.2,135.6,132.2,128.3,125.1,121.9,113.2$, 24.8 .

$\mathrm{N}$-(o-tolyl)acetamide (I 8 / II 8) [57]. White solid, m.p. 108-109 ${ }^{\circ} \mathrm{C}, 74.1 \mathrm{mg}, 99 \%$, Rf = 0.49 (Hex-EtOAc 6:4). ${ }^{1} \mathrm{HNMR}$ $\left(500 \mathrm{MHz}, \mathrm{CDCl}_{3}\right): \delta 7.71(\mathrm{~d}, J=8.0 \mathrm{~Hz}, 1 \mathrm{H}), 7.21(\mathrm{t}, J=8.0 \mathrm{~Hz}, 1 \mathrm{H}), 7.10(\mathrm{t}, J=7.5 \mathrm{~Hz}, 1 \mathrm{H}), 2.30(\mathrm{~s}, 3 \mathrm{H}), 2.19(\mathrm{~s}, 3 \mathrm{H})$. ${ }^{13} \mathrm{CNMR}\left(175 \mathrm{MHz}, \mathrm{CDCl}_{3}\right): \delta$ 168.6, 135.6, 130.5, 129.7, 126.7, 125.4, 123.7, 24.2, 17.8.

$\mathrm{N}$-(2-Nitrophenyl)acetamide (I 9 / II 9) [58]. Yellow solid, m.p. 93-94 ${ }^{\circ} \mathrm{C}, 29 \mathrm{mg}, 98 \%, \mathrm{Rf}=0.48$ (Hex-EtOAc 5:5). ${ }^{1} \mathrm{HNMR}\left(400 \mathrm{MHz}, \mathrm{CDCl}_{3}\right): \delta 10.33(\mathrm{br} \mathrm{s}, 1 \mathrm{H}, \mathrm{NH}), 8.77$ (d, $\left.J=8.4 \mathrm{~Hz}, 1 \mathrm{H}\right), 8.21(\mathrm{~d}, J=8.4 \mathrm{~Hz}, 1 \mathrm{H}), 7.65(\mathrm{t}, J=7.8 \mathrm{~Hz}$, $1 \mathrm{H}), 7.18(\mathrm{t}, J=7.8 \mathrm{~Hz}, 1 \mathrm{H}), 2.30(\mathrm{~s}, 3 \mathrm{H}) .{ }^{13} \mathrm{CNMR}\left(75 \mathrm{MHz}, \mathrm{CDCl}_{3}\right): \delta 169.1,136.4,136.0,134.9,125.8,123.3,122.2$, 25.7 .

$N$-(3-Fluorophenyl)acetamide (I 10 / II 10) [59]. White solid, m.p. 80-82 ${ }^{\circ} \mathrm{C}, 143.8 \mathrm{mg}, 99 \%$, Rf $=0.46$ (Hex-EtOAc 5:5). ${ }^{1} \mathrm{HNMR}\left(300 \mathrm{MHz}\right.$, acetone- $\left.d_{6}\right): \delta 9.32(\mathrm{~s}, 1 \mathrm{H}), 7.68(\mathrm{dd}, J=13.8,2.1 \mathrm{~Hz}, 1 \mathrm{H}), 7.29(\mathrm{~m}, 2 \mathrm{H}), 6.79(\mathrm{~m}, 1 \mathrm{H}), 2.08(\mathrm{~s}, 3 \mathrm{H})$. ${ }^{13} \mathrm{CNMR}\left(75 \mathrm{MHz}\right.$, DMSO- $\left.d_{6}\right): \delta 169.3,162.8\left({ }^{1} J_{\mathrm{CF}}=239.2 \mathrm{~Hz}\right), 141.6,130.9\left({ }^{3} J_{\mathrm{CF}}=10.2 \mathrm{~Hz}\right), 115.2,110.0\left({ }^{2} J_{\mathrm{CF}}=20.4 \mathrm{~Hz}\right)$, $106.3\left({ }^{2} J_{\mathrm{CF}}=27.2 \mathrm{~Hz}\right), 24.7$.

$\mathrm{N}$-(3-Chlorophenyl)acetamide (I 11 / II 11) [60]. White solid, m.p. 74-75 ${ }^{\circ} \mathrm{C}, 165.62 \mathrm{mg}, 99 \%$, Rf = 0.50 (Hex-EtOAc 5:5). ${ }^{1} \mathrm{HNMR}\left(300 \mathrm{MHz}, \mathrm{DMSO}-d_{6}\right): \delta 10.11(\mathrm{~s}, 1 \mathrm{H}), 7.81(\mathrm{t}, J=2.1 \mathrm{~Hz}, 1 \mathrm{H}), 7.41(\mathrm{ddd}, J=8.4,2.1,1.2 \mathrm{~Hz}, 1 \mathrm{H}), 7.29(\mathrm{t}, J=$ $8.1 \mathrm{~Hz}, 1 \mathrm{H}), 7.06$ (ddd, $J=8.1,2.1,1.2 \mathrm{~Hz}, 1 \mathrm{H}), 2.05$ (s, $3 \mathrm{H}) .{ }^{13} \mathrm{CNMR}\left(75 \mathrm{MHz}, \mathrm{DMSO}-d_{6}\right): \delta 168.6,140.7133 .1,130.4$, $122.7,118.5,117.2,24.0$.

$N$-(3-Bromophenyl)acetamide (I 12 / II 12) [61]. White solid, m.p. 85-86 ${ }^{\circ} \mathrm{C}, 199.28 \mathrm{mg}, 98 \%$, Rf $=0.53$ (Hex-EtOAc 5:5). ${ }^{1} \mathrm{HNMR}\left(300 \mathrm{MHz}\right.$, DMSO- $\left.d_{6}\right): \delta 8.18(\mathrm{~s}, 1 \mathrm{H}), 7.79(\mathrm{~s}, 1 \mathrm{H}), 7.41(\mathrm{~d}, J=7.5 \mathrm{~Hz}, 1 \mathrm{H}), 7.13-7.24(\mathrm{~m}, 2 \mathrm{H}), 2.18(\mathrm{~s}, 3 \mathrm{H})$. ${ }^{13} \mathrm{CNMR}\left(75 \mathrm{MHz}, \mathrm{DMSO}-d_{6}\right): \delta 169.0,138.2,130.2,127.2,123.1,122.6,118.5,24.4$.

$\mathrm{N}$-(3-Iodophenyl)acetamide (I 13 / II 13) [62]. White solid, m.p. 116-118 ${ }^{\circ} \mathrm{C}, 255.68 \mathrm{mg}, 99 \%$, Rf = 0.51 (Hex-EtOAc 5:5). ${ }^{1} \mathrm{HNMR}\left(300 \mathrm{MHz}\right.$; DMSO- $\left.d_{6}\right): \delta 9.51(\mathrm{~s}, 1 \mathrm{H}) ; 7.96(\mathrm{~s}, 1 \mathrm{H}), 7.45(\mathrm{~d}, J=8.0 \mathrm{~Hz}, 1 \mathrm{H}), 7.25(\mathrm{~d}, J=8.0 \mathrm{~Hz}, 1 \mathrm{H}), 6.91(\mathrm{t}, J=$ $8.1 \mathrm{~Hz}, 1 \mathrm{H}), 2.03(\mathrm{~s}, 3 \mathrm{H}) .{ }^{13} \mathrm{CNMR}\left(75 \mathrm{MHz}\right.$, DMSO- $\left.d_{6}\right): \delta$ 168.3, 139.6, 131.4, 129.6, 127.4, 118.0, 93.3, 23.6.

\section{Results and Discussion}

In order to achieve the projected reactions, we used: acetic anhydride I, acetyl chloride II, trifluoroacetic anhydride III, 2,2-dimethylpropionic anhydride IV, benzoic anhydride $\mathbf{V}$, and benzoyl chloride VI as acylating reagents. We focused especially on the acetylation process because it is the most used in synthetic procedures. The obtained results for these reactions at the beginning in this communication are presented, and then the results with III-VI with selected substrates are shown. All reaction temperatures were selected near to the corresponding boiling point for each acylation reagent at the atmospheric pressure of the experimental work $\left(100^{\circ} \mathrm{C}\right.$ for I and IV-VI; $47^{\circ} \mathrm{C}$ for II and $35^{\circ} \mathrm{C}$ for III).

For all reactions, the used relation of amines and alcohols with the acylating reagents was 1:1.4 equivalents, because in this relation the yields are slightly improved compared with relation 1:1 and other relations probed. This behavior is according to T.-S. Li and A.-X. Li report. [51]

The first set of aliphatic amines with diverse energy sources was irradiated using $\mathbf{I}$ or $\mathbf{I I}$ and the results are shown in Tables 1-2. Notably, in all reactions, SIUI irradiated reactions gave the best yields and, as the main result, with shorter reaction times. Although in both results reaction times and yields of acetylated product are similar for irradiation SIUI with I and II, the reaction temperature and more reactivity are important differences when II is used.

These results stimulated us to perform more evaluations using other substrates. Therefore, in Tables 4-7 the presented results only correspond for the use of II. (All results using both I and II in Electronic Supporting Information file are presented). 
Table 1. Acetylation of cyclohexylamine with I or II.

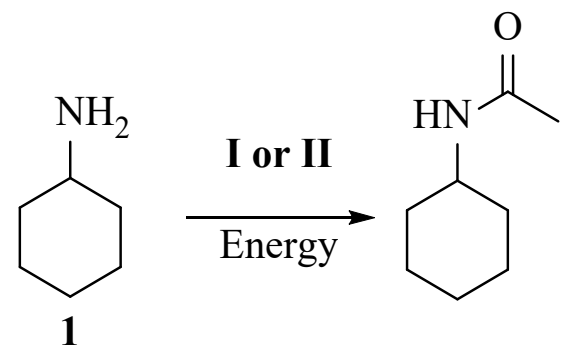

\begin{tabular}{|c|c|c|c|c|}
\hline \multirow{2}{*}{ Compound } & $\begin{array}{c}\text { SIUI }^{\mathbf{a}, \mathbf{b}} \\
\text { Time }(\min ) / \text { Yield } \\
(\%)\end{array}$ & $\begin{array}{c}\text { Thermal }^{\mathbf{a}, \mathbf{b}} \\
\text { Time }(\min ) / \text { Yield } \\
(\%)\end{array}$ & $\begin{array}{c}\text { IR }^{\mathbf{a}, \mathbf{b}} \\
\text { Time (min) / Yield } \\
(\%)\end{array}$ & $\begin{array}{c}\text { US }^{\mathbf{a}, \mathbf{b}} \\
\text { Time }(\mathrm{min}) / \text { Yield } \\
(\%)\end{array}$ \\
\hline I 1 & $0.91 / 96$ & $15 / 91$ & $20 / 90$ & $24 / 78$ \\
\hline II 1 & $0.91 / 99$ & $15 / 93$ & $20 / 94$ & $24 / 86$ \\
\hline Average & $\mathbf{0 . 9 1 / 9 7 . 5}$ & $\mathbf{1 5} / \mathbf{9 2}$ & $\mathbf{2 0} / \mathbf{9 2}$ & $\mathbf{2 4 / 8 2}$ \\
\hline
\end{tabular}

Reactions at: ${ }^{\mathrm{a}} 100{ }^{\circ} \mathrm{C}$ with I. ${ }^{\mathrm{b}} 47^{\circ} \mathrm{C}$ with II.

Table 2. Acetylation of benzylamine and $N$-methylbenzilamine with I or II.

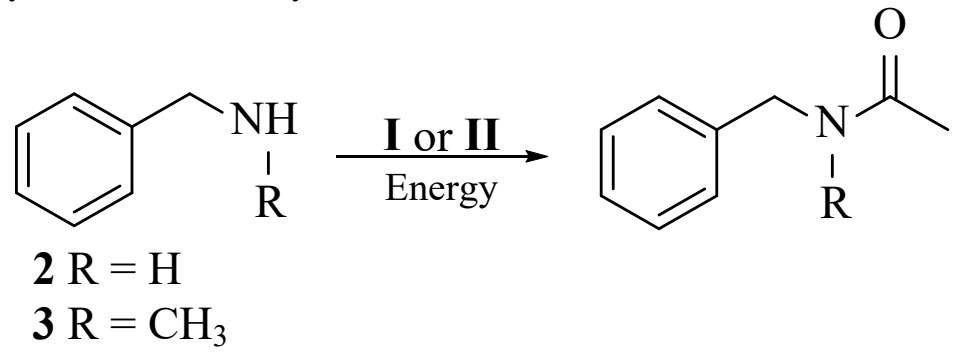

\begin{tabular}{|c|c|c|c|c|}
\hline \multirow{2}{*}{ Compound } & $\begin{array}{c}\text { SIUI }^{\mathbf{a}, \mathbf{b}} \\
\text { Time (min) / Yield (\%) }\end{array}$ & $\begin{array}{c}\text { Thermal }^{\mathbf{a}, \mathbf{b}} \\
\text { Time }(\min ) / \text { Yield } \\
(\%)\end{array}$ & $\begin{array}{c}\text { IR }^{\mathbf{a}, \mathbf{b}} \\
\text { Time (min) / Yield } \\
(\%)\end{array}$ & $\begin{array}{c}\text { US }^{\mathbf{a}, \mathbf{b}} \\
\text { Time }(\mathrm{min}) / \text { Yield } \\
(\%)\end{array}$ \\
\hline I 2 & $0.66 / 98$ & $45 / 89$ & $26 / 94$ & $46 / 88$ \\
\hline II 2 & $0.58 / 99$ & $30 / 92$ & $30 / 95$ & $54 / 90$ \\
\hline I 3 & $0.80 / 95$ & $55 / 89$ & $35 / 93$ & $56 / 84$ \\
\hline II 3 & $0.72 / 98$ & $40 / 91$ & $40 / 95$ & $60 / 87$ \\
\hline Average & $\mathbf{0 . 6 9} / \mathbf{9 7 . 5}$ & $\mathbf{4 2 . 5} / \mathbf{9 0 . 2 5}$ & $\mathbf{3 2 . 7 5} / \mathbf{9 4 . 2 5}$ & $\mathbf{5 4 . 0} / \mathbf{8 7 . 2 5}$ \\
\hline
\end{tabular}

Reactions at: ${ }^{\mathrm{a}} 100{ }^{\circ} \mathrm{C}$ with I. ${ }^{\mathrm{b}} 47^{\circ} \mathrm{C}$ with II.

For the acetylation reaction of a series of anilines, Table 3, at the same used reaction conditions, the results obtained in Table 4 are shown. 
Table 3. Selected anilines used for acetylation reaction.

\begin{tabular}{|c|c|c|c|c|}
\hline Compound & $\mathbf{R}^{1}$ & $\mathbf{R}^{2}$ & $\mathbf{R}^{3}$ & $\mathbf{R}^{4}$ \\
\hline 4 & $\mathrm{H}$ & $\mathrm{H}$ & $\mathrm{H}$ & $\mathrm{H}$ \\
\hline 5 & $\mathrm{CH}_{3}$ & $\mathrm{H}$ & $\mathrm{H}$ & $\mathrm{H}$ \\
\hline 6 & $\mathrm{Ph}$ & $\mathrm{H}$ & $\mathrm{H}$ & $\mathrm{H}$ \\
\hline 7 & $\mathrm{H}$ & $\mathrm{Br}$ & $\mathrm{H}$ & $\mathrm{H}$ \\
\hline 8 & $\mathrm{H}$ & $\mathrm{CH}_{3}$ & $\mathrm{H}$ & $\mathrm{H}$ \\
\hline 9 & $\mathrm{H}$ & $\mathrm{NO}_{2}$ & $\mathrm{H}$ & $\mathrm{H}$ \\
\hline 10 & $\mathrm{H}$ & $\mathrm{H}$ & $\mathrm{F}$ & $\mathrm{H}$ \\
\hline 11 & $\mathrm{H}$ & $\mathrm{H}$ & $\mathrm{Cl}$ & $\mathrm{H}$ \\
\hline 12 & $\mathrm{H}$ & $\mathrm{H}$ & $\mathrm{Br}$ & $\mathrm{H}$ \\
\hline 13 & $\mathrm{H}$ & $\mathrm{H}$ & $\mathrm{I}$ & $\mathrm{H}$ \\
\hline 14 & $\mathrm{H}$ & $\mathrm{H}$ & $\mathrm{H}$ & $\mathrm{F}$ \\
\hline 15 & $\mathrm{H}$ & $\mathrm{H}$ & $\mathrm{H}$ & $\mathrm{Cl}$ \\
\hline 16 & $\mathrm{H}$ & $\mathrm{H}$ & $\mathrm{H}$ & $\mathrm{Br}$ \\
\hline 17 & $\mathrm{H}$ & $\mathrm{H}$ & $\mathrm{H}$ & $\mathrm{CH}_{3}$ \\
\hline 18 & $\mathrm{H}$ & $\mathrm{H}$ & $\mathrm{H}$ & $\mathrm{NO}_{2}$ \\
\hline 19 & $\mathrm{H}$ & $\mathrm{H}$ & $\mathrm{H}$ & $\mathrm{COMe}$ \\
\hline 20 & $\mathrm{H}$ & $\mathrm{H}$ & $\mathrm{H}$ & $\mathrm{OCH}_{3}$ \\
\hline 21 & $\mathrm{H}$ & $\mathrm{H}$ & $\mathrm{H}$ & $\mathrm{NH}-\mathrm{Ph}$ \\
\hline 22 & $\mathrm{H}$ & $\mathrm{H}$ & $\mathrm{H}$ & $\mathrm{N}=\mathrm{N}-\mathrm{Ph}$ \\
\hline
\end{tabular}

Table 4. Acetylation of anilines with II.<smiles>[R]Nc1ccc([R4])c([R])c1[R]</smiles>

4-22

\begin{tabular}{ccccc}
\hline Compound & $\begin{array}{c}\text { SIUI } \\
\text { Time (min) } \\
\text { Yield (\%) }\end{array}$ & $\begin{array}{c}\text { Thermal }^{\mathbf{a}} \\
\text { Time (min) } \\
\text { Yield (\%) }\end{array}$ & $\begin{array}{c}\text { IR }^{\mathbf{a}} \\
\text { Time }(\mathrm{min}) / \\
\text { Yield }(\%)\end{array}$ & $\begin{array}{c}\text { US }^{\mathbf{a}} \\
\text { Time }(\min ) / \\
\text { Yield }(\%)\end{array}$ \\
\hline II 4 & $0.66 / 97$ & $19 / 95$ & $15 / 94$ & $18 / 96$ \\
II 5 & $1.00 / 95$ & $20 / 95$ & $16 / 95$ & $20 / 95$ \\
II 6 & $2.00 / 94$ & $17 / 96$ & $19 / 97$ & $30 / 92$ \\
II 7 & $1.00 / 97$ & $60 / 95$ & $15 / 99$ & $27 / 94$ \\
II 8 & $1.20 / 99$ & $47 / 95$ & $16 / 96$ & $23 / 94$ \\
II 9 & $1.50 / 98$ & $30 / 98$ & $15 / 97$ & $22 / 95$ \\
II 10 & $0.91 / 99$ & $20 / 96$ & $16 / 98$ & $16 / 93$ \\
II 11 & $0.92 / 99$ & $16 / 96$ & $27 / 98$ & $19 / 94$ \\
II 12 & $1.25 / 98$ & $18 / 94$ & $23 / 96$ & $18 / 91$ \\
II 13 & $0.76 / 99$ & $19 / 95$ & $15 / 97$ & $20 / 93$ \\
II 14 & $0.85 / 98$ & $20 / 98$ & $16 / 99$ & $20 / 94$ \\
II 15 & $0.88 / 99$ & $17 / 95$ & $19 / 98$ & $20 / 91$ \\
II 16 & $1.00 / 99$ & $60 / 98$ & $15 / 99$ & $30 / 95$ \\
II 17 & $0.83 / 98$ & $47 / 96$ & $16 / 98$ & $27 / 94$ \\
II 18 & $1.50 / 99$ & $30 / 96$ & $15 / 99$ & $23 / 94$
\end{tabular}




$\begin{array}{cc}\text { II } 19 & 1.10 / 99 \\ \text { II } 20 & 0.83 / 99 \\ \text { II } 21 & 1.25 / 99 \\ \text { II } 22 & 0.91 / 98 \\ \text { Average } & \mathbf{1 . 0 7} / \mathbf{9 8 . 0 5}\end{array}$

${ }^{\text {a }}$ Reactions at $47{ }^{\circ} \mathrm{C}$.

$$
\begin{aligned}
20 & \text { / } 99 \\
16 & / 97 \\
20 & / 98 \\
15 & / 95 \\
\mathbf{2 6 . 9 0} & / 96.15
\end{aligned}
$$

$16 / 97$

$27 / 98$

$22 / 93$

$23 / 97$

$16 / 95$

$20 / 96$

$19 / 94$

$24 / 95$

$18.10 / 97.26$

21.79 / 93.79

In most cases, SIUI irradiation gives slightly better reaction yields, but in all reactions, it required shorter reaction times as the main advantage of this methodology. Besides, for either electro-withdrawing or electron-releasing substituent in either position on the aromatic ring, there is no great influence on the obtained results.

For the naphthylamines 23-24, similar behavior regarding shorter reaction times for SIUI energy was observed,

\begin{tabular}{|c|c|c|c|c|}
\hline Compound & $\begin{array}{c}\text { SIUI }^{\mathbf{a}} \\
\text { Time (min) / } \\
\text { Yield (\%) }\end{array}$ & $\begin{array}{c}\text { Thermala }^{\mathbf{a}} \\
\text { Time (min) / } \\
\text { Yield (\%) }\end{array}$ & $\begin{array}{c}\mathbf{I R}^{\mathbf{a}} \\
\text { Time (min) / Yield } \\
(\%)\end{array}$ & $\begin{array}{c}\mathbf{U S}^{\mathbf{a}} \\
\text { Time (min) / } \\
\text { Yield (\%) }\end{array}$ \\
\hline II 23 & $2.5 / 92$ & $40 / 89$ & $15 / 92$ & $33 / 91$ \\
\hline II 24 & $3.0 / 91$ & $30 / 92$ & $12 / 94$ & $30 / 92$ \\
\hline Average & $2.75 / .91 .5$ & $35.0 / 90.50$ & $13.50 / 93.0$ & $31.50 / 91.5$ \\
\hline
\end{tabular}
Table 5. For II 24, although the reaction yields are slightly higher with both thermal and IR energies than SIUI energy, the reaction times of these two energies are $1000 \%$ and $400 \%$ higher than SIUI, respectively.

Table 5. Acetylation of naphthyl amines 23-24.

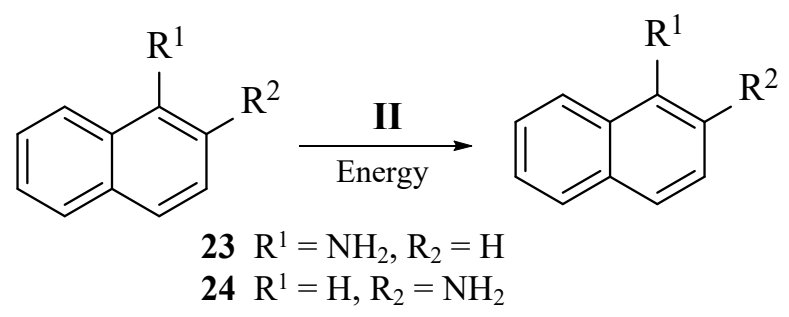

On the other hand, we evaluated the acetylation reaction of a series of alcohols, Table 6. From this Table, it is important to note the higher reaction times and the minor reaction yields for all employed energy sources compared with the reactions of amines, because of the minor nucleophilic character of the oxygen atom in alcohols than nitrogen atom in amines. Besides, is important to note that the reaction times for Thermal, IR, and US are now greater than SIUI energy in the order of $164 \%, 114 \%$, and $184 \%$, respectively. 
Table 6. Acetylation of alcohols with II.

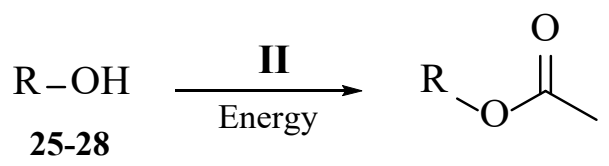

\begin{tabular}{|c|c|c|c|c|}
\hline Compound & $\begin{array}{c}\text { SIUI }^{\mathbf{a}} \\
\text { Time }(\min ) / \text { Yield } \\
(\%)\end{array}$ & $\begin{array}{c}\text { Thermal }^{\mathbf{a}} \\
\text { Time (min) / } \\
\text { Yield (\%) }\end{array}$ & $\begin{array}{c}\text { IR }^{\mathbf{a}} \\
\text { Time }(\min ) / \\
\text { Yield }(\%)\end{array}$ & $\begin{array}{c}\mathbf{U S}^{\mathbf{a}} \\
\text { Time }(\min ) / \\
\text { Yield }(\%)\end{array}$ \\
\hline \multicolumn{5}{|l|}{ Benzyl alcohol } \\
\hline $\begin{array}{c}\text { II } 25 \\
\text { Diphenyl methanol }\end{array}$ & $18 / 92$ & $50 / 87$ & $30 / 91$ & $50 / 86$ \\
\hline $\begin{array}{c}\text { II } 26 \\
\text { Phenol }\end{array}$ & $33 / 88$ & $50 / 85$ & $40 / 84$ & $70 / 76$ \\
\hline II 27 & $50 / 57$ & $85 / 63$ & $50 / 72$ & $80 / 47$ \\
\hline \multicolumn{5}{|l|}{ 2-Naphthol } \\
\hline II 28 & $48 / 94$ & $60 / 87$ & $50 / 89$ & $75 / 82$ \\
\hline Average & $37.25 / 82.75$ & $61.25 / 80.50$ & $42.50 / 84.0$ & $68.75 / 72.75$ \\
\hline
\end{tabular}

${ }^{a}$ Reactions at $47^{\circ} \mathrm{C}$.

In the same way, for the reaction with aliphatic amino alcohols 29-30, the amide group selectively was obtained, while for compound 31, only the amide group was generated, Table 7. These results confirm the highest nucleophilic character of both amine and alcohol groups in aliphatic substrates. In addition, we can find that US energy is the softest energy employed, due in each reaction the amide group is the only obtained product. That means, the lower the applied energy, the greater the selectivity.

Table 7. Acetylation of amino alcohols with II.

\begin{tabular}{|c|c|c|c|c|}
\hline Compound & $\begin{array}{c}\text { SIUI }^{\mathbf{a}} \\
\text { Time }(\mathrm{min}) / \\
\text { Yield }(\%)\end{array}$ & $\begin{array}{c}\text { Thermal }^{\mathbf{a}} \\
\text { Time }(\min ) / \text { Yield } \\
(\%)\end{array}$ & $\begin{array}{c}\mathbf{I R}^{\mathbf{a}} \\
\text { Time }(\underset{\min )}{/} / \text { Yield } \\
(\%)\end{array}$ & $\begin{array}{c}\mathbf{U S}^{\mathbf{a}} \\
\text { Time }(\min ) / \text { Yield } \\
(\%)\end{array}$ \\
\hline $\begin{array}{c}\text { 2-Amino-1-ethanol } \\
\text { II } 29\end{array}$ & $\begin{array}{c}2.5 / 85(\mathbf{A})^{*} \\
10(\mathbf{B})^{* *}\end{array}$ & $\begin{array}{c}25 / 89(\mathrm{~A}), \\
8(\mathbf{B})\end{array}$ & $\begin{array}{c}20 / 84(\mathbf{A}) \\
10(\mathbf{B}) \\
\end{array}$ & 38 / 85 (A) \\
\hline $\begin{array}{c}\text { 3-Aminopropanol } \\
\text { II } 30 \\
\end{array}$ & $3.4 / 80(\mathbf{A}), 14(\mathbf{B})$ & $26 / 87(\mathbf{A}), 9(\mathbf{B})$ & $22 / 87(\mathbf{A}), 7(\mathbf{B})$ & $36 / 87$ (A) \\
\hline $\begin{array}{c}\text { o-Aminophenol } \\
\text { II } 31\end{array}$ & $0.91 / 99(\mathbf{A})$ & $17 / 92(\mathbf{A})$ & $20 / 95(\mathbf{A})$ & $34 / 92(\mathbf{A})$ \\
\hline Average II 29-31 & $\begin{array}{c}2.27 / 88(\mathrm{~A}) \\
12.0(\mathrm{~B})\end{array}$ & $\begin{array}{c}22.67 / 89.30(\mathrm{~A}), \\
8.50(\mathrm{~B}) \\
\end{array}$ & $\begin{array}{c}20.7 \text { (A) / } 88.7 \text { (A), } 7 \\
\text { (B) }\end{array}$ & $36.0 / 88$ (A) \\
\hline
\end{tabular}

* A: $N$-Acetylation product. ${ }^{* *} \mathbf{B}: O$-Acetylation product. ${ }^{a}$ Reactions at $47^{\circ} \mathrm{C}$.

Encouraged with the results obtained, we carried out acylation reactions using III-VI with selected amines, alcohols and amino alcohols, Tables 8-10. In order to simplify the results presentation, in these tables we only present the results with the use of SIUI irradiation because in general, these results present an equivalent trend to that mentioned above. (All results with the other energy sources are included in Electronic Supporting Information). 
Table 8. Acylation of amines, alcohols and amino alcohols with III.

\begin{tabular}{|c|c|c|c|}
\hline Compound & $\begin{array}{c}\text { SIUI }^{\mathbf{a}} \\
\text { Time (min) / } \\
\text { Yield }(\%)\end{array}$ & Compound & $\begin{array}{c}\text { SIUI }^{\mathbf{a}} \\
\text { Time }(\mathrm{min}) / \\
\text { Yield }(\%)\end{array}$ \\
\hline $\begin{array}{c}\text { Cyclohexylamine } \\
\text { III } 32 \\
\end{array}$ & $0.93 / 97$ & $\begin{array}{c}p \text {-Nitroaniline } \\
\text { III } 39\end{array}$ & $2.2 / 93$ \\
\hline $\begin{array}{c}\text { Piperidine } \\
\text { III } 33 \\
\end{array}$ & $0.96 / 91$ & $\begin{array}{c}p \text {-Methoxyaniline } \\
\text { III } 40 \\
\end{array}$ & $0.91 / 92$ \\
\hline $\begin{array}{c}\text { Morpholine } \\
\text { III } 34 \\
\end{array}$ & $0.96 / 97$ & $\begin{array}{c}\text { Benzyl alcohol } \\
\text { III } 41 \\
\end{array}$ & $50 / 60$ \\
\hline $\begin{array}{c}\text { Aniline } \\
\text { III } 35\end{array}$ & $0.58 / 98$ & $\begin{array}{c}\text { 2-Amino-1-ethanol } \\
\text { III } 42 \\
\end{array}$ & $2.5 / 95^{*}$ \\
\hline $\begin{array}{c}\text { o-Bromoaniline } \\
\text { III-36 } \\
\end{array}$ & $0.95 / 97$ & $\begin{array}{c}\text { o-Aminophenol } \\
\text { III } 43 \\
\end{array}$ & $2.5 / 95^{*}$ \\
\hline $\begin{array}{c}\text { o-Nitroaniline } \\
\text { III } 37\end{array}$ & $2.3 / 94$ & $\begin{array}{c}p \text {-Aminophenol } \\
\text { III-44 }\end{array}$ & $2.5 / 93^{*}$ \\
\hline \multirow[t]{3}{*}{$\begin{array}{c}p \text {-Chloroaniline } \\
\text { III } 38 \\
\end{array}$} & $0.92 / 95$ & Average III 32-34 & $0.95 / 95$ \\
\hline & & Average III 35-40 & $1.21 / 94.8$ \\
\hline & & Average III 43-44 & $2.5 / 94.0$ \\
\hline
\end{tabular}

* $N$-acetylated product. ${ }^{a}$ Reactions at $35{ }^{\circ} \mathrm{C}$.

Table 9. Acylation of amines and naphthol with IV.

\begin{tabular}{|c|c|c|c|}
\hline Compound & $\begin{array}{c}\text { SIUI }^{\mathbf{a}} \\
\text { Time }(\mathrm{min}) / \\
\text { Yield }(\%) \\
\end{array}$ & Compound & $\begin{array}{c}\text { SIUI }^{\mathbf{a}} \\
\text { Time }(\mathrm{min}) / \\
\text { Yield }(\%)\end{array}$ \\
\hline $\begin{array}{l}\text { 1-Octylamine } \\
\text { IV } 45\end{array}$ & $0.91 / 94$ & $\begin{array}{l}\text { p-Chloroaniline } \\
\text { IV } 54\end{array}$ & $1.4 / 95$ \\
\hline $\begin{array}{l}\text { Pyrrolidine } \\
\text { IV } 46\end{array}$ & $2.3 / 90$ & $\begin{array}{l}\text { p-Phenylaniline } \\
\text { IV } 55\end{array}$ & $1.4 / 94$ \\
\hline $\begin{array}{l}\text { Morpholine } \\
\text { IV } 47 \\
\end{array}$ & $1.8 / 86$ & $\begin{array}{l}\text { p-Acetylaniline } \\
\text { IV } 56\end{array}$ & $1.8 / 95$ \\
\hline $\begin{array}{l}\text { Benzylamine } \\
\text { IV } 48\end{array}$ & $1.1 / 88$ & $\begin{array}{l}\text { p-Anilinoaniline } \\
\text { IV } 57\end{array}$ & $1.9 / 95$ \\
\hline $\begin{array}{c}N \text {-Methylbenzylamine } \\
\text { IV } 49\end{array}$ & $2.5 / 85$ & $\begin{array}{c}\text { 2-Aminonaphthalene } \\
\text { IV } 58\end{array}$ & $3 / 92$ \\
\hline $\begin{array}{c}\text { (1-Aminoethyl)benzene } \\
\text { IV 50 } \\
\end{array}$ & $1.5 / 95$ & $\begin{array}{c}\text { 3-Aminoquinoline } \\
\text { IV } 59 \\
\end{array}$ & $7 / 94$ \\
\hline $\begin{array}{c}\text { 1,2,3,4-Tetrahydroisoquinoline } \\
\text { IV } 51\end{array}$ & $5 / 79$ & $\begin{array}{c}\text { 3-Aminopyridine } \\
\text { IV } 60\end{array}$ & $10 / 74$ \\
\hline $\begin{array}{l}\text { Aniline } \\
\text { IV } 52\end{array}$ & $0.86 / 97$ & $\begin{array}{c}\text { 2-Naphthol } \\
\text { IV } 61\end{array}$ & $17 / 82$ \\
\hline $\begin{array}{c}\text { o-Bromoaniline } \\
\text { IV } 53\end{array}$ & $1.4 / 98$ & $\begin{array}{l}\text { Average IV 45-51 } \\
\text { Average IV 52-57 } \\
\text { Average IV 58-60 }\end{array}$ & $\begin{array}{l}2.16 / 88.14 \\
1.46 / 95.70 \\
6.70 / 86.70\end{array}$ \\
\hline
\end{tabular}

\footnotetext{
${ }^{\mathrm{a}}$ Reactions at $100{ }^{\circ} \mathrm{C}$.
} 
Table 10. Acylation of amines, hydrazine and 2-aminoethanol with $\mathbf{V}$ and $\mathbf{V I}$.

\begin{tabular}{|c|c|c|c|}
\hline Compound & $\begin{array}{c}\text { SIUI }^{\mathbf{a}} \\
\text { Time (min) / } \\
\text { Yield (\%) }\end{array}$ & Compound & $\begin{array}{c}\text { SIUI }^{\mathbf{a}} \\
\text { Time (min) / } \\
\text { Yield (\%) }\end{array}$ \\
\hline $\begin{array}{c}\text { Benzylamine } \\
\text { V } 62 \\
\text { VI } 62 \\
\end{array}$ & $\begin{array}{l}0.91 / 89 \\
0.91 / 94\end{array}$ & $\begin{array}{c}\text { o-Methoxyaniline } \\
\text { V } 69 \\
\text { VI } 69 \\
\end{array}$ & $\begin{array}{l}1 / 89 \\
1 / 98\end{array}$ \\
\hline $\begin{array}{c}N \text {-Methylbenzylamine } \\
\text { V } 63 \\
\text { VI } 63\end{array}$ & $\begin{array}{l}0.91 / 84 \\
0.91 / 91\end{array}$ & $\begin{array}{c}\text { o- Nitroaniline } \\
\text { V } 70 \\
\text { VI } 70\end{array}$ & $\begin{array}{c}1 / 93 \\
0.91 / 96\end{array}$ \\
\hline $\begin{array}{c}\text { Pyrrolidine } \\
\text { V } 64 \\
\text { VI } 64\end{array}$ & $\begin{array}{l}0.91 / 70 \\
0.91 / 76\end{array}$ & $\begin{array}{c}o \text {-Methylaniline } \\
\text { V } 71 \\
\text { VI } 71\end{array}$ & $\begin{array}{l}0.91 / 92 \\
0.91 / 96\end{array}$ \\
\hline $\begin{array}{l}\text { Morpholine } \\
\text { V } 65 \\
\text { VI } 65\end{array}$ & $\begin{array}{l}0.91 / 76 \\
0.91 / 87\end{array}$ & $\begin{array}{c}\text { 2-Aminopyridine } \\
\text { V } 72 \\
\text { VI } 72\end{array}$ & $\begin{array}{l}0.91 / 65 \\
0.91 / 75\end{array}$ \\
\hline $\begin{array}{c}\text { Cyclohexylamine } \\
\text { V } 66 \\
\text { VI } 66 \\
\end{array}$ & $\begin{array}{l}0.91 / 90 \\
0.91 / 94 \\
\end{array}$ & $\begin{array}{c}\text { Hydrazine } \\
\text { V } 73 \\
\text { VI } 73 \\
\end{array}$ & $\begin{array}{l}0.91 / 82 \\
0.91 / 89 \\
\end{array}$ \\
\hline $\begin{array}{c}\text { Aniline } \\
\text { V } 67 \\
\text { VI } 67\end{array}$ & $\begin{array}{l}0.66 / 91 \\
0.66 / 96\end{array}$ & $\begin{array}{c}\text { 2-Aminoethanol } \\
\text { V } 74 \\
\text { VI } 74\end{array}$ & $\begin{array}{c}0.91 / 85(\mathbf{A})^{*} \\
0.91 / 65(\mathbf{A}), 19(\mathbf{B})^{* *}\end{array}$ \\
\hline $\begin{array}{c}o \text {-Bromoaniline } \\
\text { V } 68 \\
\text { VI } 68\end{array}$ & $\begin{array}{l}1 / 92 \\
1 / 98\end{array}$ & $\begin{array}{r}\text { Average V 62-66 } \\
\text { Average VI 62-66 } \\
\text { Average V 67-71 } \\
\text { Average VI 67-71 } \\
\text { Average V 62-71 } \\
\text { Average VI 62-71 }\end{array}$ & $\begin{array}{l}0.91 / 81.80 \\
0.91 / 88.40 \\
0.91 / 91,40 \\
0.89 / 96.80 \\
0.91 / 86.60 \\
0.90 / 92.60\end{array}$ \\
\hline
\end{tabular}

* A: $N$-Acylation product. ${ }^{* *} \mathbf{B}: O$-Acylation product. ${ }^{a}$ Reactions at $100{ }^{\circ} \mathrm{C}$.

In view of the results for amines, alcohols, and amino alcohols, it is clear that the new SIUI methodology is the best condition to achieve the corresponding acylated compound. This method can be used with certainty that the generation of good results in shorter reaction times and with good selectivity for amino alcohols will occur. Therefore, the minor use of energy for SIUI is an excellent approach to the sixth principle of green chemistry.

Affording to results, the new SIUI methodology is a good alternative compared with the other energy sources employed to obtain good to excellent reaction yields in shorter reaction times. However, it is important to mention some limitations for the SIUI method, such as alcohol acylation reactions and, in the other hand, that is necessary to improve the experimental procedure because reactions must be carried out in 1-2 minute periods to avoid projections of the reaction mixtures. We are currently making efforts in this regard.

It is known that electromagnetic infrared irradiation alters both rotational and vibrational behavior of molecules and the ultrasound energy, by the high energy level of bubble cavitation, can induce molecule excitation and particle fragmentations. It is difficult to propose how the simultaneous combination of these energies act in the presented reactions, so this synergism must be studied.

\section{Conclusions}

The acylation of anilines, alcohols and amino alcohols with the new simultaneous infrared-ultrasound irradiation method are successfully achieved with excellent reaction yields, full conversion of substrates to the corresponding acylated product and shorter reaction times compared with the other energy sources employed.

Importantly, in all presented reactions the most valorous advantage of SIUI methodology is not the general superior reaction yields, but the shorter reaction times. Therefore, the minor use of energy with SIUI apparatus is an excellent 
approach to the sixth principle of green chemistry. This new experimental synthetic methodology can be a good alternative in organic synthesis and obviously, it must be evaluated in other chemical transformations, which represents next and new challenges.

\section{Acknowledgments}

Authors want to thank to DGAPA-UNAM for the financial support to IN218515 project and Ricardo Alfredo Luna to CONACYT for the Grant 32841.

\section{References}

1. Wuts, P. G. M.; Greene, T. W., in: Greene's Protective Groups in Organic Chemistry. 4th edition. John Wiley and Sons Ed., New Jersey, 2007.

2. Petursson, S. J. Chem. 2013, 1-10. Article ID 183049.

3. Sartori, G.; Ballini, R.; Bigi, F.; Bosica, G. R.; Maggi, R.; Righi, P. Chem. Rev. 2004, 104, 199-250. DOI: 10.1021/cr0200769

4. Yadav, J. S.; Narsaiah, A. V.; Basak, A. K.; Goud, P. R.; Sreenu, D.; Nagaiah, K. J. Mol. Catal. A Chem. 2006, 255, 78-80. DOI: 10.1016/j.molcata.2006.03.066

5. Seed, M.; Brown, J. R.; Freemantle, C. Cancer Res. 1997, 57, 1625-1629.

6. Masahiko, T.; Kawano, S.; Tsuji, S.; Sawaoka, H.; Hori, R.; Du Bois, N. Cell 1998, 93, 705-716. DOI: 10.1016/S0092-8674(00)81433-6

7. Adinolfi, M.; Barone, G.; Iadonisi, A.; Schiattarella, M. Tetrahedron Lett. 2003, 44, 4661-4663. DOI: 10.1016/S0040-4039(03)01072-4

8. Habibi, D.; Rahmani, P.; Akbaripanah, Z. J. Chem. 2013, Article ID 972960.

9. Beranek, J.; Hrebabecký, H. Nucleic Acids Res. 1976, 3, 1387-1202._DOI:10.1093/nar/3.5.1387

10. Moffet, R. B.; Anderson, H. V. J. Am. Chem. Soc. 1954, 76, 747-749. DOI: 10.1021/ja01632a032

11. Rowell, R. M. Mol. Cryst. Liq. Cryst. 2004, 418, 153-164. DOI: $10.1080 / 15421400490479244$

12. Mizuno, N.; Misono, M. Chem. Rev. 1998, 98, 199-218. DOI: 10.1021/cr960401q

13. Mojtahedi, M. M.; Saeed, M.; Heravi, A. M.; Behbahani, F. K. Monatsh. Chem. 2007, 138, 95-99. DOI:10.1007/s00706-006-0564-3

14. Marwah, P.; Marwah, A.; Lardy, H. A. Tetrahedron 2003, 59, 2273-2287. DOI:10.1016/S0040-4020(03)00207-2

15. Ishihara, K.; Kubota, M.; Yamamoto, V. Synlett 1996, 3, 265-266. DOI:10.1055/s-1996-5376

16. Ishihara, K.; Nakayama, M.; Ohara, S.; Yamamoto, H. Tetrahedron 2002, 58, 8179-8188. DOI: 10.1016/S00404020(02)00966-3

17. Dhanon, M. K.; Olsen, R. K.; Ramaswamy, K. J. Org. Chem. 1982, 47, 1962-1965. DOI: 10.1021/jo00349a028

18. Carrigan, M. D.; Freiberg, D. A.; Smith, R. C.; Zerth, H. M.; Mohan, R. S. Synthesis 2001, 33, 2091-2094. DOI: 10.1002/ejoc.200300754

19. Yadav, J. S.; Narsaiah, A. V.; Basak, A. K.; Goud, P. R.; Sreenu, D.; Nagaiah, K. J. Mol. Cat. A 2006, 255, 78-80. DOI:10.1016/j.molcata.2006.03.066

20. Ishihara, K.; Kubota, M.; Kurihara, H.; Yamamoto, H. J. Org. Chem. 1996, 61, 4560-4567. DOI: $10.1021 /$ jo952237x

21. Procopiou, P. A.; Baugh, S. P. D.; Flack, S. S.; Inglis, G. G. A. J. Org. Chem. 1998, 63, 2342-2347. DOI: $10.1021 /$ jo980011z

22. Orita, A.; Tanahashi, C.; Kakuda, A. J. Org. Chem. 2001, 66, 8926-8934. DOI: 10.1021/jo0107453

23. Chakraborti, A. K.; Gulhane, R. Synlett 2003, 12, 1805-1808. DOI:10.1055/s-2003-41442

24. Yoon, H. J.; Lee, S. M.; Kim, J. H.; Cho, H. J.; Choi, J. W.; Lee, S. H. Tetrahedron Lett. 2008, 49, 3165-3168.

25. Joseph, J. K.; Jain, S. L. Mol. Cat. A 2007, 267, 108-111. DOI:10.1016/j.molcata.2006.11.026

26. Das, B.; Thirupathi, P.; Kumar, R. A.; Laxminarayana, K. Adv. Synth. Catal. 2007, 349, 2677-2683. DOI: 10.1002/adsc.200700292

27. Kumareswaran, R.; Panchamuthu, V.; Vankar, Y. D. Synlett 2000, 11, 1652-1654. DOI: 10.1055/s-2000-7925

28. Fromentin, E.; Coustard, J. M.; Guisnet, M. J. Mol. Catal. A: Chem. 2000, 159, 377-388. DOI:10.1016/S13811169(00)00225-9 
29. Guignard, C.; Pèdron, V.; Richard, F.; Jacquot, R.; Spagnol, M.; Coustard, J. M.; Pérot, G. Appl. Catal. A 2002, 234, 79-90. DOI:10.1016/S0926-860X(02)00199-0

30. Varma, R. S.; Namboodiri, V. V. Pure Appl. Chem. 2001, 73, 1309-1313.

31. Braibante, H. T. S.; Braibante, M. E. F.; Rosso, G.B.; Oriques, D. A. J. Braz. Chem. Soc. 2003, 14, 994-997. DOI:10.3998/ark.5550190.0009.c24

32. Das, S. K.; Reddy, K. A.; Krovvidi, V. L. N. R.; Mukkanti, K. Carbohydr. Res. 2005, 340, 1387-1392. DOI:10.1016/j.carres.2005.03.001

33. Gholap, A. R.; Venkatesan, K.; Daniel, T.; Lahoti, R. J.; Srinivasan, K. V. Green Chem. 2003, 5, 693-696. DOI: 10.1039/B308069B

34. Valdez-Rojas, J. E.; Ríos-Guerra, H.; Ramírez-Sánchez, A. L.; García-González, G.; Álvarez-Toledano, C.; LópezCortés, J. G.; Toscano, R. A.; Penieres-Carrillo, J. G. Can. J. Chem. 2012, 90, 567-573. DOI: 10.1139/v2012-030

35. Liu, Y.; Liu, L.; Lu, Y.; Cai, Y. Q. Monatsh. Chem. 2008, 139, 633-638. DOI:10.1007/s00706-007-0814-Z

36. Alleti, R.; Woon, S. O.; Perambuduru, M.; Afrasiabi, Z.; Sinn, E.; Reddy, V. P. Green Chem. 2005, 7, 203-206. DOI: $10.1039 / \mathrm{B} 416359 \mathrm{~A}$

37. Forsyth, S. A.; MacFarlane, D. R.; Thomson, R. J.; Von Itzstein, M. Chem. Commun. 2002, 7, 714-715. DOI:10.1039/b200306f

38. Machado, L. L.; Lemos, T. L. G.; de Mattos, M. C. Tetrahedron: Asymmetry 2008, 19, 1419-1424. DOI:10.1016/j.tetasy.2008.05.018

39. Benfatti, F.; Cardillo, G.; Gentilucci, L.; Mosconi, E.; Tolomelli, A. Tetrahedron: Asymmetry 2007, 18, 2227-2232. DOI:10.1016/j.tetasy.2007.09.002

40. Escobedo, R.; Miranda, R.; Martínez, J. Int. J. Mol. Sci. 2016, 17, 453-478. (And therein references) DOI:10.3390/ijms17040453

41. Peng, Y.; Song, G. Green Chem. 2001, 3, 302-304. DOI: 10.1039/B108878P

42. Cravotto, G.; Cintas, P. Chem. Eur. J. 2007, 13, 1902-1909. DOI: 10.1002/chem.200601845

43. L Chemat, F.; Poux, M.; Di Martino, J. L.; Berlan, J. J. Microw. Power Electromagn. Energy 1996, 31, $19-22$. DOI:10.1080/08327823.1996.11688288

44. Peng, Y.; Song, G. Green Chem. 2002, 4, 349-351. DOI: 10.1039/B201543A

45. Peng, Y.; Song, G. Green Chem. 2003, 5, 704-706. DOI: 10.1039/b310388a

46. Palmisano, G.; Bonrath, W.; Boffa, L.; Garella, D.; Barge, A.; Cravotto, G. H. Adv. Synth. Catal. 2007, 349, 23382344. DOI: 10.1002 adse.200700098

47. Rossi, D.; Urbano, M.; Carnevale, B. A.; Serra, M.; Bergamelli, F.; Iannelli, M.; Azzolina, O.; Collina, S. Synth. Commun. 2009, 39, 3254-3263. DOI:10.1080/00397910902738112

48. Shen, X. F. J. Chem. Technol. Biotechnol. 2009, 84, 1811-1817. DOI:10.1002/jctb.2250

49. Palmisano, G.; Tagliapietra, S.; Barge, A.; Binello, A.; Boffa, L.; Cravotto, G. Synlett 2007, 2041-2044. DOI: $10.1055 / \mathrm{s}-2007-984891$

50. Martina, K.; Tagliapietra, S.; Barge, A.; Cravotto, G. Top. Curr. Chem. 2016, 374-379. DOI 10.1007/s41061-016$0082-7$

51. Li, T.-S; Li, A.-X. Perkin Trans. 1998, 1, 1913-1917. DOI: 10.1039/A802051E

52. Ortega, F.; Domínguez, F. X.; Rosas, A.; Penieres, G.; López, J. G.; Ortega, M. C. Appl. Organomet. Chem. 2015, 29, 556-560. DOI: $10.1002 /$ aoc.3331

53. Maegawa, T.; Akashi, A.; Yaguchi, K.; Iwasaki, Y.; Shigetsura, M.; Monguchi, Y.; Sajiki, H. Chem. Eur. J. 2009, 15, 6953-6963. DOI: 10.1002/chem.200900361

54. Soulé, J.F.; Miyamura, H.; Kobayashi, S. J. Am. Chem. Soc. 2011, 133, 18550-18553. DOI: 10.1021/ja2080086

55. Xiang, D.; Xia, L.; Zhang, Y.; Zhang, Q.; Li, D. Synlett, 2018, 29, 1400-1404. DOI: 10.1055/s-0036-1591970

56. García, N.; García, P.; Fernández, M.A.; Rubio, R.; Pedrosa, M.R.; Arnáiz, F.J.; Sanz, R. Adv. Synth. Catal. 2012, 354, 321-327. DOI: 10.1002/adsc.201100877

57. Lee, Y.M.; Moon, M.E.; Vajpayee, V.; Filimonov, V.D.; Chi, K.W. Tetrahedron 2010, 66, 7418-7422. DOI: 10.1016/j.tet.2010.07.005

58. Dos Santos, C.M.G.; McCabe, T.; Watson, G.W.; Kruger, P.E.; Gunnlaugsson, T. J. Org. Chem. 2008, 73, 92359244. DOI: $10.1021 /$ jo8014424

59. Saikia, U.; Hussain, F.; Suri, M.; Pahari, P., Tetrahedron Lett. 2016, 57, 1158-1160. DOI: 10.1016/j.tetlet.2016.01.108

60. Sun, X.; Wang, M.; Li, P.; Zhang, X.; Wang, L., Green Chem. 2013, 15, 3289-3294. DOI: 10.1039/C3GC41260A

61. Lal, S.; Snape, T., J. Mol. Catal. B: Enzym. 2012, 83, 80-86. DOI: 10.1016/j.molcatb.2012.07.007

62. Rosenthal, A.; Melby, R.; Sandin, R.; B. J Am Chem Soc. 1952, 74, 5790-5791. DOI: 10.1021/ja01142a525 